\title{
Geometric Morphometrics to the Study of Skull Sexual Dimorphism in a Local Domestic Goat Breed
}

\section{Parés-Casanova PM*}

Department of Animal Production, School of Agrifood and Forestry Science and Engineering, University of Lleida Av. Rovira Roure 191, 25198 Lleida, Catalunya, Spain

\begin{abstract}
The existence of sexual dimorphism in the White Rasquera goat breed has long been known, with males being larger than females. However, no study so far has explored this dimorphism separately in its size and shape components. Here we analysed twenty-three skeletonized skulls from adult animals (11 males and 12 females) belonging to this breed by means of geometric morphometric (GM) methods using 7 landmarks on dorsal aspect. Differences observed between the sexes are size related, with males bigger than females. Shape is also an important component separating males from females. The difference in skull form between sexes may be interpreted in relation to extensive management styles of the animals: being under a low anthropogenic influence they tend to reinforce their natural sexual size dimorphism. This being the first known study to apply GM to a domestic goat breed, we advocate for further GM research in domestic breeds in order to have the possibility of comparing populations and easily exchanging information between research centres.
\end{abstract}

Keywords: Animal ethnology; Dorsal cranium; Neurocranium; Viscerocranium; White rasquera goat

\section{Introduction}

In recent years, functional studies incorporating geometric morphometric (GM) analyses have become increasingly common [13]. Since GM techniques have been discussed at length elsewhere [4-7], a detailed discussion is considered not necessary here.

GM analyses the relative positions of anatomical landmarks and sets of points used to approximate curves (outlines) and surfaces to quantify size and shape [8]. These techniques offer an advantage over traditional analyses of linear morphometrics in that the geometric shape space presents all various shape configurations of designated landmarks $[1,4,9]$. The geometric information of shape differences is preserved, statistical power is increased [10] and patterns can be visualized using image rendering and a variety of other diagrams [11]. In taxonomy and other fields, genetics and morphometrics can fruitfully interact as complementary tools to understand the origin of phenotypic differences [12]. The rise in publications using GM outside biology has been exponential and pays testament to the success of this set of methods but this type of analysis has not yet been extensively performed in domestic mammals.

Goats are sexually dimorphic animals, with a general pattern of males being larger than females. Most of the research on this species has been done on a purely biometric basis of linear measurements, so the animal ethnologist interested in focusing on shape, separately from size, can have difficulties on testing hypotheses about shape differences.

The White Rasquera goat breed is a racial grouping located in the Southern region of Catalonia (NE Spain). This breed is very similar to the White Celtiberica one, a population of meat aptitude bred to produce kids. Being both sexes horned, animals show a strong sexual dimorphism, as well as remarkable morphostructural variability within genders. The breed has great rusticity and is perfectly adapted to local orographic and weather conditions [13]. Until now, all morphological researches with this breed have been done at a lineal morphometric level $[13,14]$. So this research was intended to assess differences in sexspecific skull shape based on 2D GM.

\section{Material and Methods}

Twenty four skeletonized skulls from pure-bred animals belonging to the White Rasquera breed were initially sampled from a private local collection. We selected only adult specimens with known sex. To obtain comparable results across groups and across anatomical regions, an initial balanced sample of 12 males and 12 females was studied. All animals were adults (the tooth row was employed as an age criteria thus only animals with all permanent teeth erupted were selected) [15]. Care was taken to avoid specimens lacking major parts and to obtain good quality pictures so a male specimen was excluded from research and 23 skulls were finally studied.

\section{Morphological characterization}

For the analysis of differences between sexes related to size and shape of the skull, we used GM. Skulls were dorsally photographed. Image capture was performed with a tripod-mounted Nikon ${ }^{\circ}$ D70 digital camera (image resolution of 2,240 × 1,488 pixels) equipped with a Nikon AF Nikkor $28-200 \mathrm{~mm}$ telephoto lens. The focal axis of the camera was parallel to the horizontal skull plane and centred on its dorsal aspect. A ruler was used in this process (interval 1 $\mathrm{mm})$. Skulls were photographed once with the same procedure. The dorsal aspect is an interesting structure in domestic breed studies as, although anatomical landmarks are not numerous, it is where linear measurements are normally obtained in order to establish cephalic indices for ethnological classification. All images were saved on JPEG format and stored in a personal computer.

Morphometric analysis must be based on unambiguous and repeatable anatomical marks. Our chosen landmarks represented different points from the neurocranium and viscerocranium (facial skeleton) and were selected because of the ease of their location on fixed bony points; moreover they were not "masked" by the large horns that characterize this breed. Seven landmarks were collected for each

*Corresponding author: Parés-Casanova PM, Department of Animal Production School of Agrifood and Forestry Science and Engineering, University of Lleida, Av. Rovira Roure 191, 25198 Lleida, Catalunya, Spain, Tel: +34973706460, Fax +34973702874; E-mail: peremiquelp@prodan.udl.cat.

Received July 13, 2015; Accepted August 01, 2015; Published August 10, 2015

Citation: Parés-Casanova PM (2015) Geometric Morphometrics to the Study of Skull Sexual Dimorphism in a Local Domestic Goat Breed. J Fisheries Livest Prod 3: 141. doi:10.4172/2332-2608,1000141

Copyright: @ 2015 Parés-Casanova PM. This is an open-access article distributed under the terms of the Creative Commons Attribution License, which permits unrestricted use, distribution, and reproduction in any medium, provided the original author and source are credited. 
individual. In order to avoid redundant information only the right side of cranium was used (Figure 1): (1) akrokranion (medial point on the os supraoccipitale), (2) ectorbitale (point of the greatest width in os frontale), (3) facial crest, (4) facial tuberosity, (5) premaxilla (widest part), (6) nasion (the intersection of the frontal bone and two nasal bones), and (7) prosthion (the most anterior point in the midline on the alveolar process of the maxilla). Two additional landmarks spaced 40 $\mathrm{mm}$ apart were also included for scaling (Table 1).

We used the software TpsUtility 1.50 [16] to organize the images. Landmarks were digitized using TpsDig 2.04 [17]. Shape variables were obtained as linear combinations of the original landmark coordinates after standardizing size and removing artifactual variation

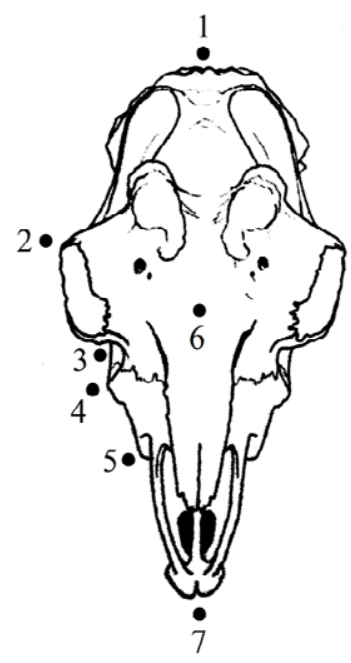

Figure 1: Landmarks used in the analyses (numbers correspond to definitions in text). Chosen landmarks represented different points from the neurocranium and viscerocranium (facial skeleton) and were selected because of the ease of their location on fixed bony points; moreover they were not "masked" by the large horns that characterize this breed. In order to avoid redundant information only the right side of cranium was used.

\begin{tabular}{|c|c|c|c|}
\hline PC & Eigenvalues & \% Variance & Cumulative $\%$ \\
\hline 1 & 0.00097662 & 34.246 & 34.246 \\
\hline 2 & 0.00083573 & 29.306 & 63.551 \\
\hline 3 & 0.00029333 & 10.286 & 73.837 \\
\hline 4 & 0.00023396 & 8.204 & 82.041 \\
\hline 5 & 0.00013243 & 4.644 & 86.685 \\
\hline 6 & 0.00012426 & 4.357 & 91.042 \\
\hline 7 & 0.00007830 & 2.746 & 93.788 \\
\hline 8 & 0.00006535 & 2.291 & 96.079 \\
\hline 10 & 0.00005350 & 1.876 & 97.955 \\
\hline 11 & 0.00002215 & 0.777 & 98.732 \\
\hline 12 & 0.00001125 & 0.394 & 99.126 \\
\hline 13 & 0.00000912 & 0.320 & 99.446 \\
\hline 14 & 0.00000667 & 0.234 & 99.680 \\
\hline 15 & 0.00000465 & 0.163 & 99.843 \\
\hline 16 & 0.00000185 & 0.065 & 99.908 \\
\hline 17 & 0.00000144 & 0.050 & 99.958 \\
\hline 18 & 0.00000071 & 0.025 & 99.983 \\
\hline & 0.00000049 & 0.017 & 100.000 \\
\hline
\end{tabular}

Table 1: Eigenvalues from a Principal Component Analysis treating the skulls $(n=24)$ as the object of interest and the lineal measurements as the variables. None of the Principal Components (PC) presented an eigenvalue $>1$. due to different positions of the specimens during the process of data collection (generalized Procrustes analysis). Landmark positions were converted to scaled $\mathrm{x}$ and $\mathrm{y}$ coordinates using CoordGen6f [18]. CoordGen6f was also used to translate and rotate images. We saved centroid size (CS) to be used as a measurement independent of shape [19], which is the square root of the summed distances between each landmark coordinate and the centroid of the landmark configuration [20]. Shape differences were visualized with deformation grids, where one object (reference) is deformed into another (target) and shape features can be described in terms of deformation grids depicting the differences between objects [11]. The thin plate spline algorithm was used to compute the deformation grid with the least bending energy between the reference and target landmark configurations.

Landmarks were digitized twice. Profile plots of the landmark coordinates were used to detect landmarks with relatively large variations between repetitions. The two repeats tend to cluster together; bit correlation between the matrices of Procrustes distances from each of the repetitions was very high (Mantel test $r=0.723, p=0$ ). Digitizing error was considered, therefore, negligible.

To assess differences in shape between sexes, principal-components analysis (PCA) on the covariance matrix and discriminant function analysis (DFA) were performed. PCA was used to display variation within a sample, to characterize the main features of shape variation and as an ordination method. Shape variables were regressed onto the first two PCA axes to illustrate shape changes along them. DFA was used to separate sex groups. It provides an ordination that maximizes the separation of the group means relative to the variation within groups and emphasizes the degree of separation of the groups and not, as in canonical variate analysis, the relative arrangement of multiple groups. Accordingly, more emphasis is placed on the probability of correct and incorrect classification of observations. In order to compare sizes (CS) between sexes, values were log-transformed. The Mann-Whitney $U$ test was used for significant differences in $\log$ CS medians between sexes and the Kolmogorov-Smirnov D test for overall equal distribution of two sex samples (for both cases using CS, as it does not assume normal distribution). Multivariate ANOVA (MANOVA) was done in order to compare shape between sexes. As size affects the skull and can inflate measures, we explored the effect of allometry by computing a multivariate regression of shape on log CS. To adjust for size-shape differences we repeated the analyses using the residuals of the multivariate regression CS was considered the independent variable. This regression allowed us to correct for the effects of size on shape (allometry). The Mann-Whitney $U$ test was used to test for significant differences in allometric slopes and intercepts between sexes and the MANOVA to test differences in regression scores between sexes. Using the residuals from a regression of shape on centroid size was the method to correct for the effects of allometry. Significance was accepted at $\mathrm{p} 0.05$ level.

\section{Numerical statistical analysis}

Data were analysed using MorphoJ [21] and PAST-"Paleontological Statistics Software Package for Education and Data Analysis" [22] software.

\section{Ethics statement}

No specific permits were required for this study as it did not involve neither slaughtering animals nor collecting endangered or protected organic parts. 


\section{Results}

The first component, PC1, accounted for $41.7 \%$ of the variation. Viscerocranium saggital points (landmarks 6 and 7) made the largest contribution to the total variance. The second component, PC2, accounted for $33.5 \%$ of the variation and was a measure of viscerocranium points, too (Figure 2).

The $\log$ CS was different between the sexes $(U=31, p<0.05)$, being larger in male skulls than in females (Figure 3 ), and also their distribution was different $(D=0.659, p<0.05)$. Apart from size, there appeared to be shape differences between the sexes (Wilks $\Lambda=0.130$, df $1=14$, df $2=8$, $\mathrm{p}<0.05)$. Inter-individual allometry was significant $(\mathrm{p}=0.02)$ although it explained a small percentage (12.7\%) of shape differences according to size (Figure 4). When size effect was already removed significant differences were still present between the sexes, so the differences here observed were not only in shape. Post-hoc comparisons between the
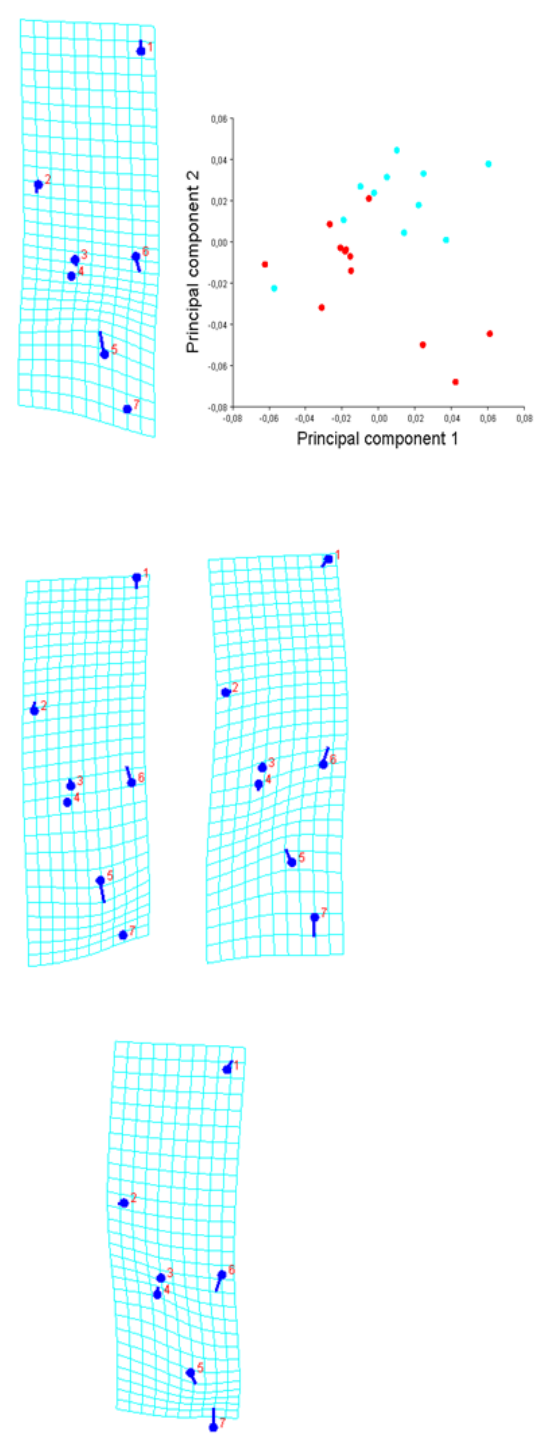

Figure 2: Principal Component Analysis for PC1 and PC2. PC1, accounted for $41.7 \%$ of the variation. Viscerocraniumsaggital points (landmarks 6 and 7) made the largest contribution to the total variance. The second component, PC2, accounted for $33.5 \%$ of the variation and was a measure of viscerocranium points (landmarks 5 and 6 ).

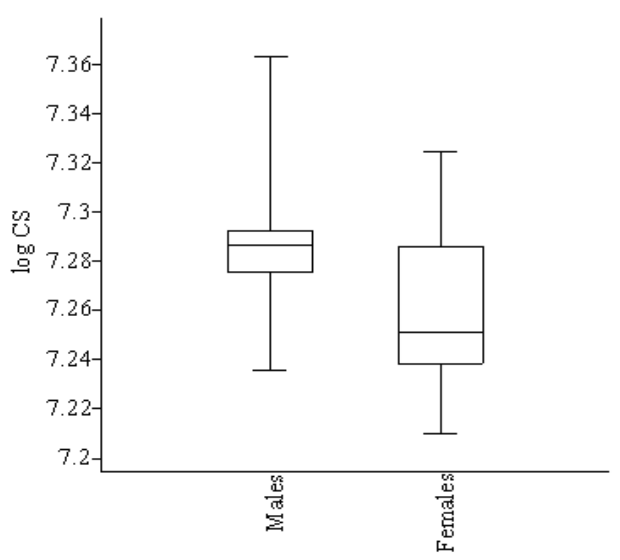

Figure 3: Boxplot for centroid size for males and females. The 25-75 percent quartiles are drawn using a box. The median is shown with a horizontal line inside each one. The minimal and maximal values are shown with short horizontal lines ("whiskers"). Log centroid size (CS) was not different between the sexes.

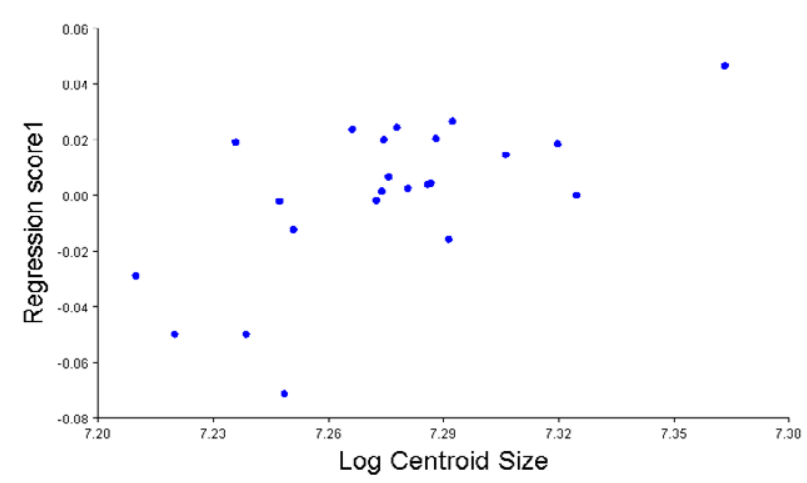

Figure 4: Multivariate regression of the shape variables on log centroid size Inter-individual allometry was significant $(p=0.02)$ although it explained a small percentage $(12.7 \%)$ of shape differences according to size.

sexes found neither differences in their allometric slopes as regressed onto head shape $(\mathrm{U}=86.5, \mathrm{p}=0.613)$ nor in the intercepts $(\mathrm{U}=97.0$, $\mathrm{p}=0.981)$, but regression scores for both sexes were different $(\mathrm{p}<0.05)$ being larger for males than for females.

\section{Discussion}

We can draw some preliminary conclusions as to the level of significance of shape and size variations in White Rasquera adult animals. First of all, the very small error demonstrated that the proposed landmarks can be located with precision and images were acquired with accuracy despite the lack of flatness of the dorsal cranial plane. The analyses of the univariate size measurements and multivariate shape data clearly show that the breed is sexually dimorphic for head size and shape, having males a larger skull. Allometry reveals that shape differences are not simply due to allometry. The anatomical separation of the skull into viscerocranium and neurocranium is due to functional separations within both regions (i.e. the facial skeletal morphology develops and responds to visual, olfactory and masticatory soft tissues and functions, whereas the neurocranium mainly responds to CNS growth and vascularization while functioning to protect the brain) and being the face where most sexual changes appear.

Sexual dimorphism (SD) is a widespread phenomenon in different 
animal taxa, including the subfamily of goats and sheep (Caprinae), which belongs to the most dimorphic mammalian groups [23]. Rensch's rule describes the pattern of sexual dimorphism, claiming that larger species generally exhibits higher male to female body size ratio [23, 24]. Agreement with Rensch's rule has been manifested by slope of the allometric relationship between male and female skull size exceeding one. The existence of SD in the White Rasquera breed has long been known, males being larger than females [13], perhaps due to the fact that being under a low anthropogenic influence they tend to reinforce their natural sexual size dimorphism. However, no study so far had explored this dimorphism separately in its size and shape components and focused on skull. Here, we exploited both size and shape to gain insights into skull SD for his breed. An interesting side issue to our study is that if size is excluded from the analysis, individuals may be grouped for sex.

This is the first known study to apply GM to a domestic goat breed, so comparisons of our data to other published sources are not possible. We advocate for further GM research in domestic breeds in order to have the possibility of comparing populations and easily exchanging information between research centres.

\section{Acknowledgement}

Author must thank to the local farms where sampling of skulls were kindly allowed. Too to Josué Sabaté, who was responsible to collect, clean and photograph the specimens. We thank finally the anonymous reviewers whose comments helped to enhance the overall quality of the manuscript.

\section{References}

1. Rohlf FJ, Loy A, Corti M (1996) Morphometric analysis of old world talpidae (Mammalia, Insectivora) using partial-warp scores. Sys Biol 45: 344-362.

2. Rosas A, Bastir M (2004) Geometric morphometric analysis of allometric variation in the mandibular morphology of the hominids of Atapuerca, Sima de los Huesos site. Anat Rec A Discov Mol Cell Evol Biol 278: 551-560.

3. Oettlé AC, Pretorius E, Steyn M (2005) Geometric morphometric analysis of mandibular ramus flexure. See comment in PubMed Commons below Am J Phys Anthropol 128: 623-629.

4. Bookstein FL (1996) Combining the tools of geometric morphometrics. In: Marcus LF, Corti M, Loy A, Naylor GJP, Slice DE (eds) Advances in morphometrics. Plenum, New York.

5. Monteiro LR, Reis SF (1999) Princípios de morfometria geométrica
6. Zelditch ML, Swiderski DL, Sheets HD, Fink WL (2004) Geometric morphometrics for biologists: A primer. San Diego, CA. Elsevier Academic.

7. Slice DE (2005) Modern morphometrics in physical anthropology. New York: Kluwer Academic.

8. Jensen RJ (2003) The conundrum of morphometrics. Taxon 52: 663-671.

9. Rohlf FJ (1998) On applications of geometric morphometrics to studies of ontogeny and phylogeny. Syst Biol 47: 147-158.

10. James Rohlf F, Marcus LF (1993) A revolution morphometrics. See comment in PubMed Commons below Trends Ecol Evol 8: 129-132.

11. Adams DC, Slice DE, Rohlf FJ (2004) Geometric morphometrics: Ten years of progress following the 'revolution'. It J Zool 71: 05-16.

12. Klingenberg CP (2010) Evolution and development of shape: integrating quantitative approaches. See comment in PubMed Commons below Nat Rev Genet 11: 623-635.

13. Carné S, Roig N, Jordana J (2007) The Blanca de Rasquera Goat breed: morphological and phaneroptical characterization. Arch Zootec 56: 319-330.

14. Parés PM, Sabaté J, Jordana J (2010) Multivariate morphometric analysis of caprine skulls. III Congresso Iberico de Paleontologia, Lisboa.

15. Dyce KM, Sack WO, Wensing CJG (1996) Veterinary Anatomy. WB Saunders Company, Philadelphia, New York, London.

16. Rohlf FJ (2012) Tps Utility Program, ver. 1.50. Department of Ecology and Evolution, Stony Brook, State University of New York, New York.

17. Rohlf FJ (2005) TpsDig, digitize landmarks and outlines, ver. 2.04. Department of Ecology and Evolution, Stony Brook, State University of New York, New York.

18. Sheets HD (2003) IMP-integrated morphometrics package. Department of Physics, Canisius College, Buffalo, New York.

19. Bookstein FL (1991) Morphometric tools for landmark data: geometry and biology. Cambridge Univ. Press, New York.

20. Dryden IL, Mardia KV (1998) Statistical shape analysis. John Wiley and Sons, Chichester.

21. Klingenberg CP (2011) MorphoJ: an integrated software package for geometric morphometrics. Mol Ecol Resour 11: 353-357.

22. Hammer $\varnothing$, Harper DAT, Ryan PD (2001) PAST: Paleontological Statistics Software Package for Education and Data Analysis. Palaeontol Elec 4: 1-2.

23. Polák J, Frynta D (2009) Sexual size dimorphism in domestic goats, sheep, and their wild relatives. Biol J Linnean Soc 98: 872-883.

24. Frynta D, Baudyaova J, Hradcova P, Faltusova K, Kratochva L (2012) Allometry of sexual size dimorphism in domestic dog. PLoS One 7: e46125.
Citation: Parés-Casanova PM (2015) Geometric Morphometrics to the Study of Skull Sexual Dimorphism in a Local Domestic Goat Breed. J Fisheries Livest Prod 3: 141. doi:10.4172/2332-2608.1000141

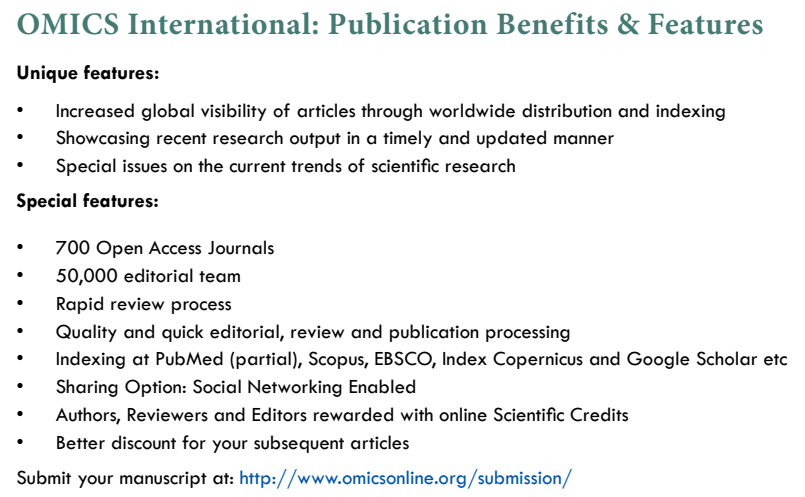

